\title{
Study the Influence of a Gap between the Wing and Slotted Flap over the Aerodynamic Characteristics of Ultra-Light Aircraft Wing Airfoil
}

\author{
Cvetelina Velkova ${ }^{1}$, Michael Todorov ${ }^{1}$ and Guillaume Durand ${ }^{2}$ \\ 1 Department of Mechanics, Faculty of Transport, Technical University, Sofia 1000, Bulgaria \\ 2. ECAM, Strasbourg-Europe, 2 rue de Madrid - 67300 Schiltigheim, France
}

Received: March 05, 2015 / Accepted: March 20, 2015 / Published: May 25, 2015.

\begin{abstract}
The purpose of the study is to assess what the influence of the distance of the gap is between the wing and slotted flap on the aerodynamic characteristics of ultra-light aircraft wing when the flap is retracted. It has been elected numerical approach to the study and it is been realized through applied numerical model of the wing airfoil NACA 2412 for three different lengths of slotted gap size, whose length is expressed as percentages of the airfoil chord. The code ANSYS FLUENT has been applied, as it has been determined RANS (Reynolds-averaged Navier-Stokes) equations and DES (detached-eddy simulation) turbulent model has been used.
\end{abstract}

Key words: Aerodynamics, wing airfoil, slotted flap, gap, numerical approach.

\section{Introduction}

As it is summarized by Dam [1], the problems of high-lift aerodynamics have been studied since the early years of aviation. Gunther, et al. [2] quotes, "The wings of commercial aircrafts must generate a tremendous amount of lift during take-off and landing in order to reduce ground speeds and runway lengths". It can be concluded that employing typical high-lift systems for different kinds of aircrafts can fulfill that requirements. But like it is stated by Gunther, et al. [2] instead of complex multi-element high-lift devices for improvement of lift aerodynamics of ultra-light aircraft wing, a single slotted flap is desirable.

As indicated by Young [3], when a portion of the rear of a wing can be rotated to leave a well-defined slot between it and the rest of the wing, then it is referred to slotted flap. The main function of flaps is to be used as tool for aerodynamics improvements. In

\footnotetext{
Corresponding author: Cvetelina Velkova, Ph.D., research field: computation fluid dynamics. E-mail: cvvelkova@tu-sofia.bg.
}

consequence, the slotted flap was then adopted in order to provide more lift to reduce the stalling speed and decrease the drag to increase the gliding angle [3].

In order to give perspective to the present review of slotted flap and the influence of the gap, some short overview is in order. The slotted flap is a trailing-edge device which is separated from the main wing element by very small gaps [4]. The representation of the mechanical design for slotted flap is given in Fig. $1 b$. Citing from Ref. [5], the point where the airfoil is first cut away to form the slot on the lower surface is called the slot entry, i.e. gap is the distance between airfoil and the flap (Fig. 1b).

The aerodynamic characteristics of single slotted flap were admirably reviewed by Forster and Irwin [6]. Since a slotted flap is an effective device for increasing the maximum lift coefficient $C_{L}$ and decreasing the drag coefficient $C_{D}$ provided by the flow through the slot, the important design parameter that should be better studied is optimal distance of the gap between the wing and slotted flap. However, unless the 


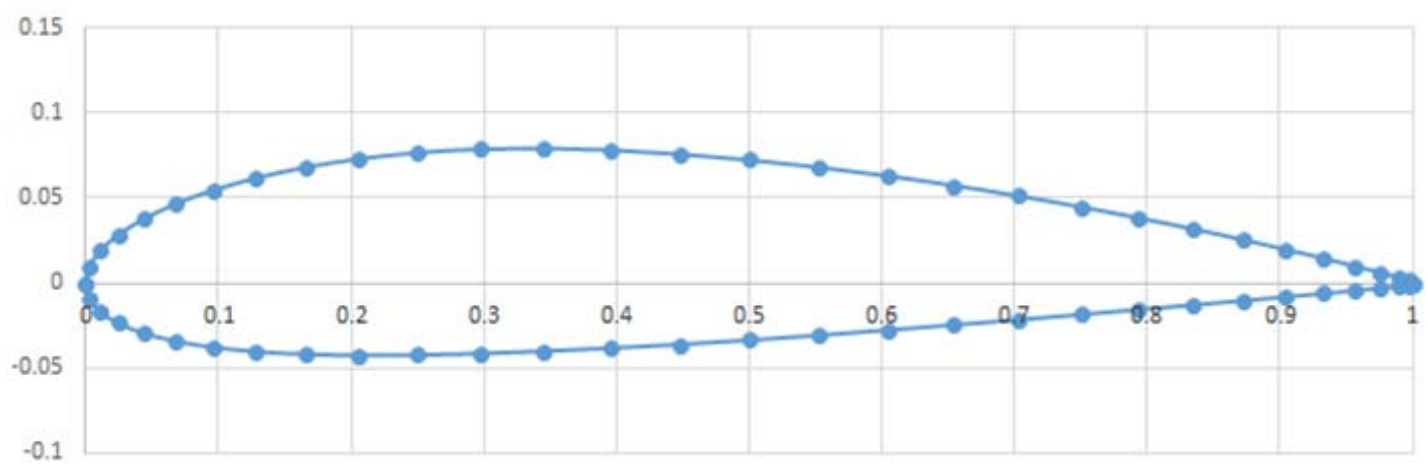

(a)

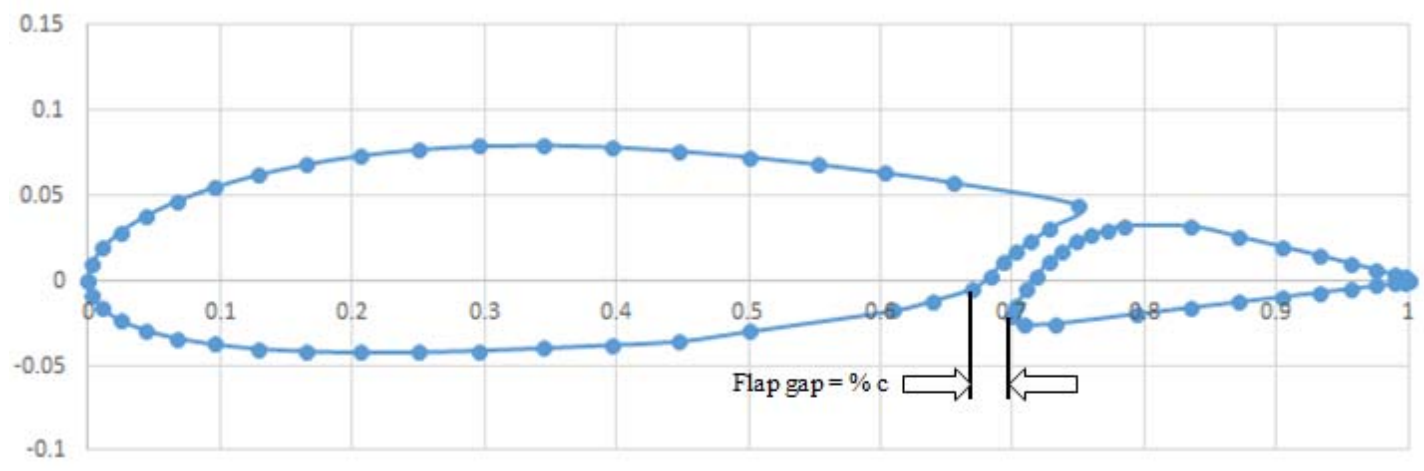

(b)

Fig. 1 (a) pure wing airfoil NACA 2412 and (b) configuration with slotted flap.

slotted flap is shown to provide higher maximum $C_{L}$ than any other devices (plain flaps and split flaps), the best length of gap is not so well defined [5, 7].

The reason for the choice of the optimal gap size is in respect of high lift capabilities and low drag contribution of the wing. The optimization of the gap size between the wing and slotted flap is a difficult problem because of its dependence on a few parameters: airfoil shape, wing camber, nature of the flow, boundary layer and Reynold number $(R e)$. Because of the fact that the slotted flap is high-lift system, it has been characterized by complex flow physics [4]. That is why it has been cited Smith in Ref. [4], and the effect of slotted flap can be achieved by properly designed gaps.

Since, slotted flap is dominated by viscous effects as boundary layer transition, flow separation, etc. For optimizing the gap size, the only way is the numerical approach. The proper numerical simulation of the two dimensional flow field around the configuration of single slotted flap can be achieved by using the full Navier-Stokes equations with a suitable turbulent viscosity model.

In the last years of the successful application of 2D CFD (computational fluid dynamics), Navier-Stokes code is playing a strategic role in the prediction of the aerodynamic performance of the wing [8]. But the computation of the flow over a multi-element wing in lift configuration and optimization of the gap size remains, however, one of the most difficult problems encountered in CFD. The main goal of the study is the development of efficient numerical approach for aerodynamic analysis and optimization.

As summarized by Cumming and Scott [4], a good review of computational capabilities for simulating of the flow around lift system (wing with slotted flap) was recently conducted by Rumsey and Ying. They concluded that there were three main areas of improvements required for the prediction of lift system aerodynamics: (1) including unsteady effects; 
(2) improving turbulent models; and (3) improving modeling fidelity. This study extends the previous two-dimensional, lift CFD research in two ways suggested by Rumsey and Ying: by adding unsteady effects of the computations, and by using a higher-order hybrid DES (detached-eddy simulation) $k-\omega$ SST (shear stress transport) turbulent model.

The DES model is a method for predicting turbulence in CFD computations, which combines a RANS (Reynolds-averaged Navier-Stokes) method in the boundary layer with an LES (large eddy simulation) in the free shear flow. DES turbulent model gives a good accuracy of the flow field because it solves an additional equation for turbulent Reynolds number in an SST version. Using DES turbulence model is effective because it gives better visualization of flow field, the unsteady separation flow and vortex shedding. The reason for that study is investigating the influence of the gap size between the ultra-light aircraft wing and slotted flap by optimization tools.

Using the most suitable turbulent model for numerical simulation of the flow field around the whole lift configuration in 2D gives the ability to evaluate better aerodynamic characteristics of that type of configuration. Applied more accurate numerical approach flow simulation around that type of lift system is a prerequisite for giving the more precise evaluation of the slotted gap size, in consequence improving the aerodynamics of ultra-light aircraft wings.

\section{Method of Solution}

As summarized by Brezillon, et al. [8], the aerodynamics around a wing in high-lift configuration is one of the most complex flows occurring around the airplane. Except for typical boundary layer regions near the walls the multi-element wing also of great importance are the re-circulation areas in the cut-outs, mixing of the boundary layers and the wakes of proceeding elements and secondary flow located to the gap between the wing and slotted flap [8]. Moreover, since the ultra-light aircraft wing operates in different range of angles of attack $\alpha$, the flow separation is most likely to occur in the wing and flap upper section. Therefore, the flow in lift configuration is dominated by viscous effects. As it has been confirmed by many studies in this area, the most appropriate numerical method to correctly capture all these flow features is CFD code. The last one is capable to solve compressible RANS equations for 2D flow.

In order to capture all of these critical flow features, a grid of high quality is required [8]. For this study, to be able to appreciate the influence of slotted gap size on the aerodynamic characteristics of the wing, thick boundary layer had to be used in numerical model. Hence, a thick layer of thin cells has been generated orthogonal to the wall of the wing airfoil and slotted flap in numerical CFD model.

\subsection{Numerical Model Representation}

A CFD 2D wing airfoil model domain and suitable grid mesh are used. For the purpose of that study, numerical model of the ultra-light aircraft wing equipped with slotted flap is applied to airfoil NACA 2412. As the justification of the chosen airfoil can point out the fact that NACA 2412 has been used in multiple applications, one example being the wing of section of Cessna 150-aircraft [9]. To fulfill the purpose of studying the influence of gap size between the wing and slotted flap the prepared numerical computations were made for three different cases of the gap size. The choice of the gap size for the cases is based on preliminary research done in this area for that study. The parameter of the gap size is defined as percentages of airfoil chord $-c$. The values of the gap size for three different cases of the numerical computations are: (1) $1.8 c$; (2) $2.8 c$; and (3) $3.2 c$. It is worth to mention that in that case the wing flap deflection angle is ignored, in other words in that case model of wing is equipped with slotted flap without deflection. This case is typical for horizontal cruise flight of the aircraft. 
Several numerical simulation of the flow around pure wing airfoil in 2D and the numerical flow simulation around the configuration of wing with slotted flap have been performed. Type of the pure wing airfoil and configuration with slotted flap is given in Figs $1 \mathrm{a}$ and $1 \mathrm{~b}$.

The initial data for the airfoil NACA 2412 are taken from NACA database [10], and slotted flap coordinates take into account [6]. In this study, the simulation of the unsteady flow field around wing with slotted flap configuration has been realized using FLUENT solver.

\subsection{Grid Generation}

The applied methodology for the execution of the objective of that study - the distance of the gap size is hybrid unstructured grid approach. Hybrid approach clearly simplifies meshing procedure and permits to consider more complex configuration [8]. The advantages of this approach lie in its simplification in grid generation and can be achieved with so-called overset techniques.
Grid structure and details are shown in Fig. 2 for the generated typical grid mesh used in the numerical computations. The computational domain around a model has radius of curvature around the airfoil $14 c$ and the length of numerical domain after the configuration: wing-slotted flap $25 c$. The wing airfoil is located on $14 c$ from the entrance of the computational domain. The mesh density i.e., number of nodes at the upper and lower section of the airfoil and slotted flap is greater in order to provide a better visualization of flow field near the surfaces, where is possible acceleration of the flow.

The grid is quad map structure mesh, and it has 247,331 cells and 251,116 nodes. The grid for the all three cases with different slotted gap size is strongly compressed close to the wing airfoil contour, particularly in the gap regions. The latter is done in order to better intercept mixing shear layers and flow separation which is characters effect for the high-lift configuration, as in this study. Compressing of the grid mesh close to the contour of the configuration is made by size function tool of the Gambit, which is

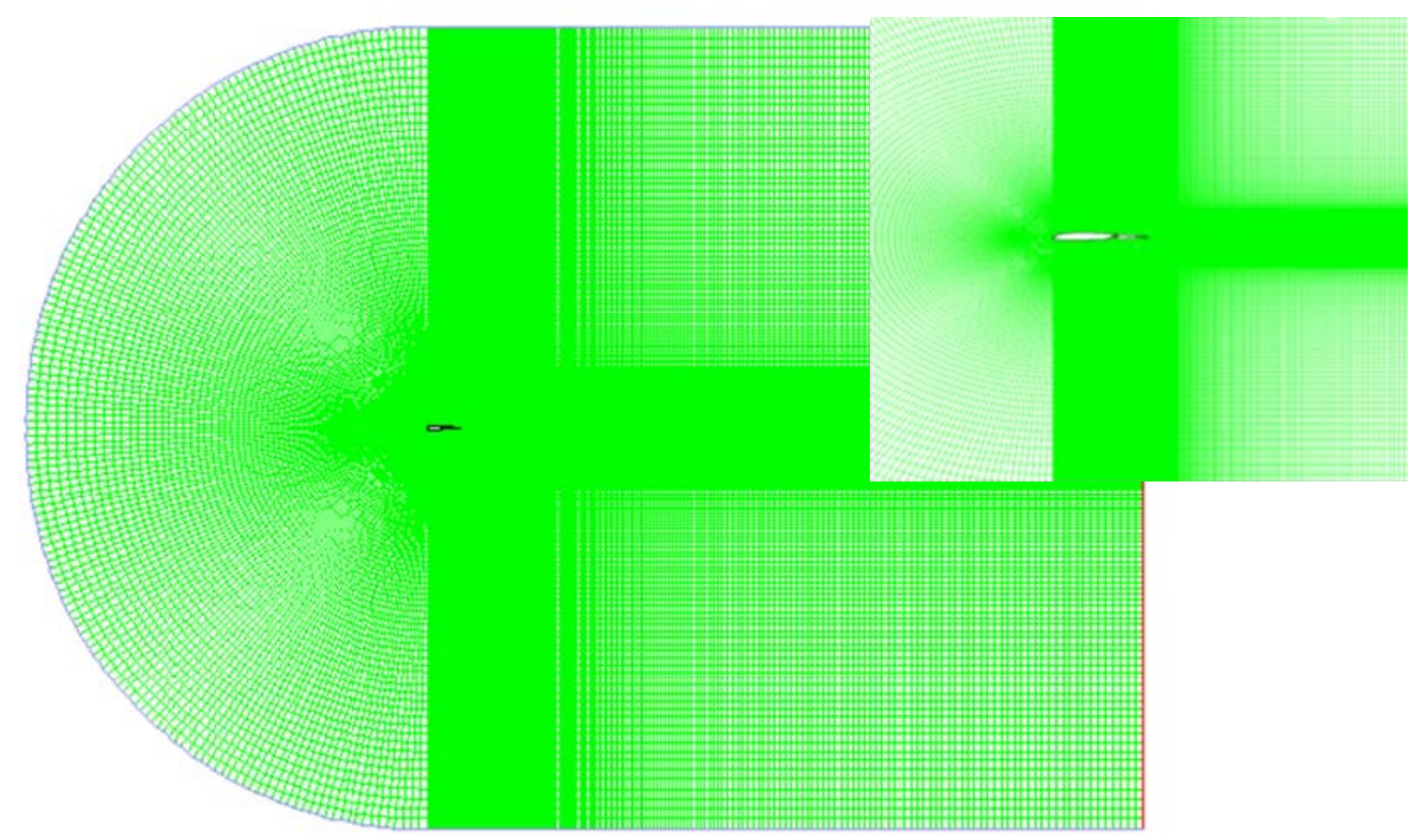

Fig. 2 The mesh over the airfoil domain and grid near the configuration-wing-slotted flap. 
part from FLUENT solver. The quality of the mesh plays a significant role in the accuracy and stability of the numerical computations. Regardless of the type of mesh over the computational domain, checking the quality of mesh is essential. The important indicator of mesh quality that FLUENT allows to check is Wall Yplus, which is $8.9 \mathrm{e}+01$ for the contour of the airfoil and slotted flap, which is sufficient enough to ensure better visualization of the unsteady flow field in the solution process.

The method of solution is iterative. The fluid CFD 2D wing airfoil model with slotted flap clarifies the unsteady flow field around the whole lift configuration by calculating the aerodynamic characteristic of wing airfoil wake. The last action gives the ability to conclude the influence of the gap size, consequently for better evaluation and improvement in the complex aerodynamics of such type of lift configuration. The solution is transient process because of the unsteady flow field, and the characteristics are calculated to each time step.

Time step $\Delta t=1 \times 10^{-3}$ is used, i.e., over 1,000 number of time-steps $(\sim 1.0 \mathrm{~s})$. Because of the unsteady flow field around wing airfoil slotted flap configuration and $R e$ number corresponds with the free stream velocity $V_{\infty}=30 \mathrm{~m} / \mathrm{s} V_{\infty}=50 \mathrm{~m} / \mathrm{s}$. The value of the free stream velocity used for the numerical calculation is complying with the objective of that study-ultra-light aircraft wing.

The SIMPLE algorithm is employed for the pressure/velocity coupling and solution of the momentum equations is obtained using a bounded central differencing scheme. Unsteady computations are made with a second order dual time stepping algorithm, which is stable for large time steps and thereby decreases the computational time. Various turbulent models and mesh procedures exist and have successfully been used. Referring to the large number of investigation in that area made until this days, it can be concluded that DES $k-\omega$ SST approach is quite suitable because of its ability to deliver good results especially for low $R e$ number for aerodynamic characteristic, pressure and skin friction distribution, while saving the computational time and memory.

\section{Results and Analysis}

The obtained results are presented in Figs. 3-5. DES $k-\omega$ SST turbulence model used for calculation is a hybrid model. The $k-\omega$ SST turbulence model is used to model the flow near the airfoil and to capture better the flow in the regions of the slotted gap size. It is worth to mention that the obtained numerical results for wing with slotted flap configuration are for unsteady flow and are for three different cases of the gap size.

Fig. 3 represents the made comparison between experimental static lift coefficient $C_{L}$ of the pure wing airfoil NACA 2412 taken from Ref. [11], numerical results with DES for $C_{L}$ for pure wing airfoil and the

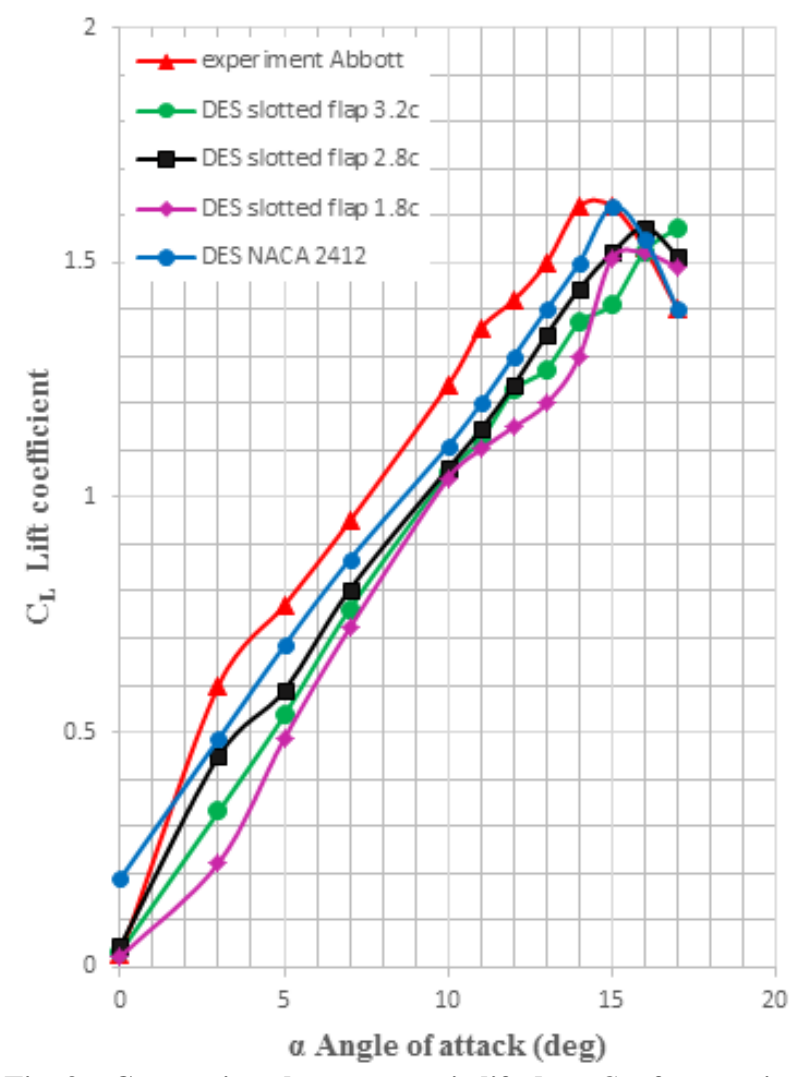

Fig. 3 Comparison between static lift data $C_{L}$ of pure wing airfoil NACA 2412 with DES, experiment and for the configuration wing-slotted flap according to three different cases of the gap size. 

Characteristics of Ultra-Light Aircraft Wing Airfoil

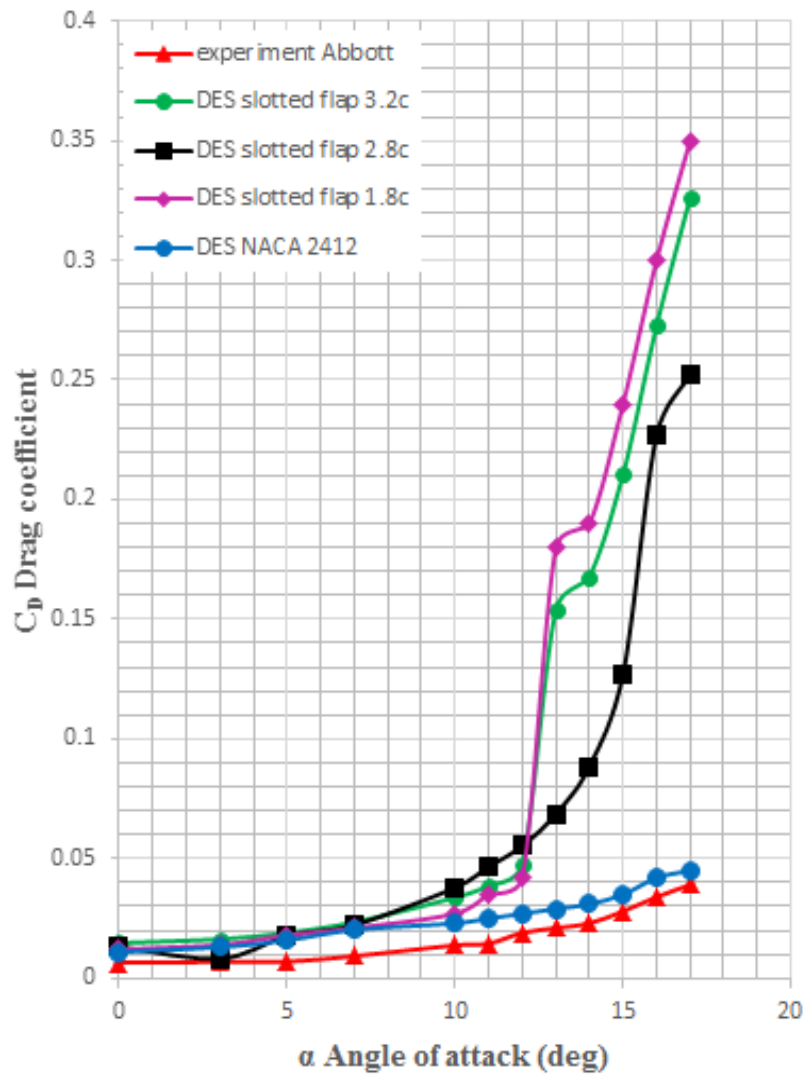

Fig. 4 Comparison between drag coefficient $C_{D}$ for pure wing airfoil NACA 2412 with DES, experiment and for configuration wing-slotted flap according to gap sizes.

obtained numerical results for wing with slotted flap configuration for three different cases of the gap size.

Fig. 4 gives the representation for made comparison between experimental data for $C_{D}$ of pure wing airfoil NACA 2412 taken from Ref. [11], numerical results with DES for pure wing airfoil and the configuration with slotted flap for three cases according to the gap size.

The obtained numerical results for aerodynamic characteristics of $C_{L}$ and $C_{D}$ are approximated well with the experimental data (Figs. 3 and 4). Also from the polar of $C_{L}$ versus, it can be observed that DES $k-\omega$ SST turbulence model gives good estimation of stall angle. It can be observed from Fig. 3, where obtained numerical results from the calculations show that the stall angle for the configuration: Wing-slotted flap is higher approximately $1^{\circ}$ than the actual stall angle on the experimental curve of $C_{L}$ (Fig. 3).

To fulfill the purpose of that study, and hence to improve the aerodynamics of ultra-light aircraft wing, our task in analyzing the obtained results was to define which of the three different cases of the gap size gives the best aerodynamic characteristics. On the stage of results analysis, our goal was to conclude which of the chosen cases for the varying with the gap size gives the higher lift coefficient $C_{L}$ according to the increasing of the drag coefficient $C_{D}$. Figs. 3 and 4 show that in the proposed configuration of the wing with retracted flap, the lift coefficient $C_{L}$ does not differ significantly throughout range of angles of attack $\alpha$. Unlike the lift coefficient $C_{L}$, drag coefficient $C_{D}$ starts significantly to differ at $\alpha=12^{\circ}$ (for NACA 2412 critical angle of attack $\alpha=13^{\circ}$ ). At these angles of attack $\alpha$, the highest $C_{D}$ is obtained at a distance of slotted gap size $1.8 c$, and the lower $C_{D}$ is observed at case $2.8 c$ (Figs. 3 and 4).

Therefore, the optimal value of distance of gap is expected to be higher than $2.8 c$ and lower than $3.2 c$.

Referring on the above considerations, it can be made a general conclusion that even though in the horizontal flight when the flap is not diverted, influence of gap size is sufficient to higher angles of attack. The last statement is confirmed by the fact that in the three different cases of the slotted flap size, the predicted stall angle is $16^{\circ}$, which is $1^{\circ}$ more than the actual stall angle given in the experimental curve of $C_{L}$ for pure wing as shown in Fig. 3 .

Fig. 5 illustrated velocity magnitudes for pure wing and configuration: wing-slotted flap. The influence of the slotted gap size and the accuracy of the applied numerical DES $k$ - $\omega$ SST model are confirmed by the shown results taken from the numerical simulations in CFD code. Observing Fig. 5, it is clear that the velocity of the upper section of the wing airfoil is faster than the velocity on the lower section, reaching the gap there can be observed dilution of the flow field and then reaching the upper flap surface the flow is fully stalled and the velocity is decreased. The last effect is confirmed by the observation of velocity magnitude in case of pure wing and the other three 


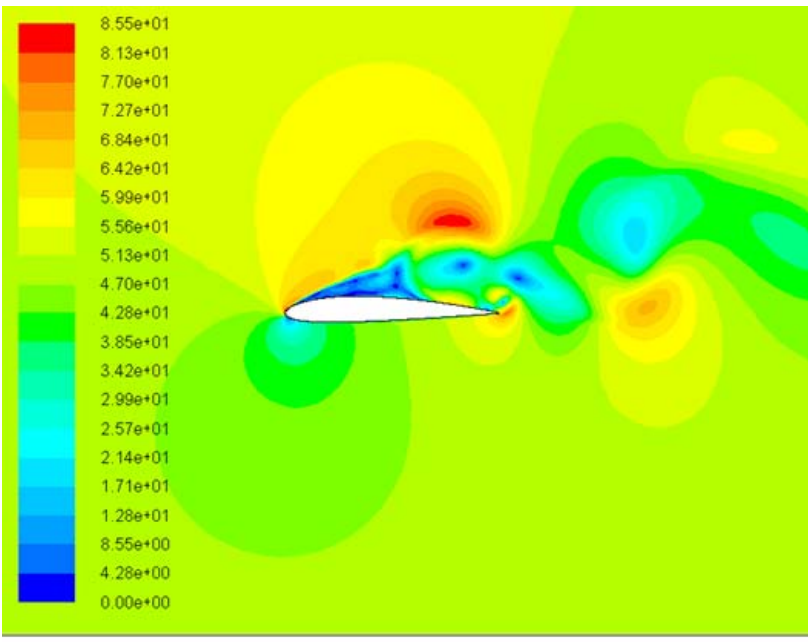

Contours of Velocity Magnitude $(\mathrm{m} / \mathrm{s})($ Time $=1.0000 \mathrm{e}+00)$

(a)

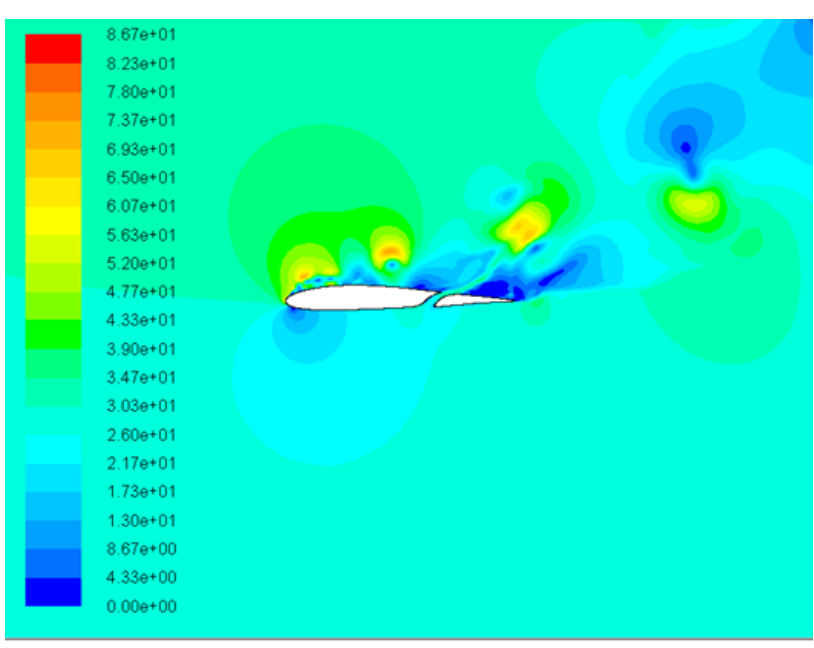

Contours of Velocity Magnitude $(\mathrm{m} / \mathrm{s})($ Time $=1.0000 \mathrm{e}+00)$

(c)

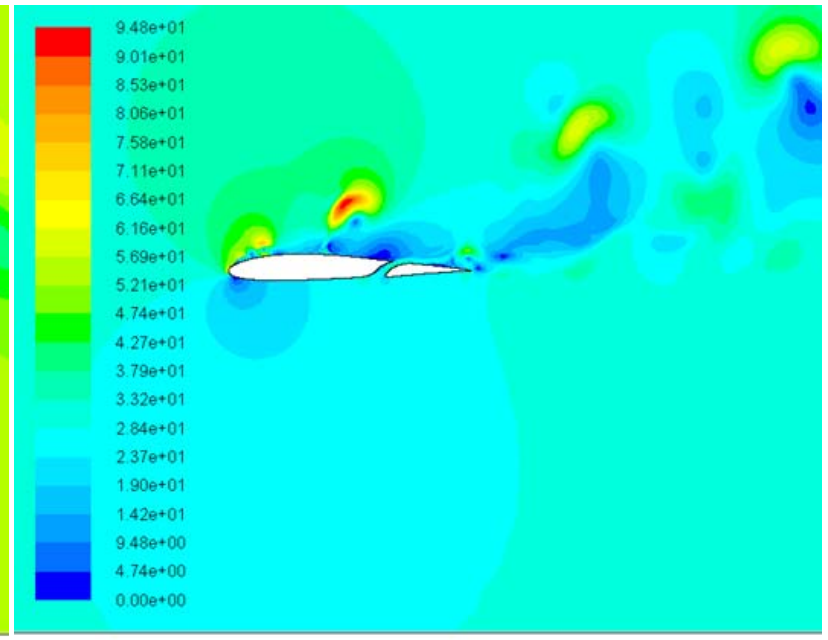

Contours of Velocity Magnitude $(\mathrm{m} / \mathrm{s})($ Time $=1.0000 \mathrm{e}+00)$

(b)

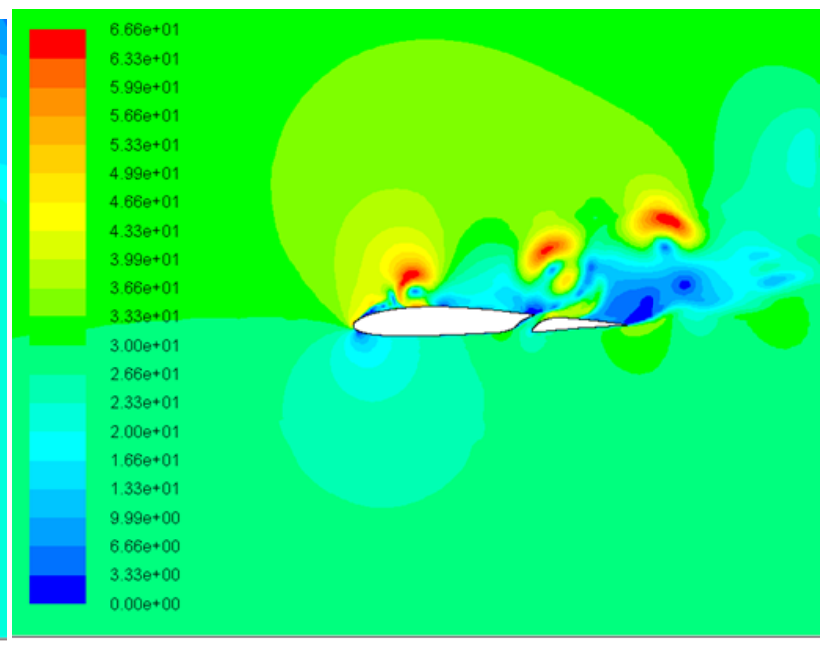

Contours of Velocity Magnitude $(\mathrm{m} / \mathrm{s})($ Time $=1.0000 \mathrm{e}+00)$

(d)

Fig. 5 (a): velocity magnitude at $\alpha=16^{\circ}$, pure wing; (b) velocity magnitude at $\alpha=16^{\circ}$, gap 2.8c; (c) velocity magnitude at $\alpha$ $=18^{\circ}$, gap 2.8c; and (d) velocity magnitude at $\alpha=16^{\circ}$, gap 3.2c for two-dimensional DES $k-\omega$ SST modeling.

cases in configuration with slotted flap. The numerical results for the velocity in Fig. 5 confirmed the influence of the gap size, as can be seen the velocity is higher in case $2.8 c$ for $16^{\circ}$. Normally, if it was taken into account the slotted flap deflection increasing the angles of attack, the flow must be accelerated with increasing the flap deflection, and the whole configuration had to confirm its function for creation of more lift. But on the basis of this case in that study, the only conclusion that can be made is the fact that the suggested numerical model namely the DES $k-\omega$ SST model for modeling the flow field around the configuration wing-slotted flap works and gives relatively satisfactory results.

\section{Conclusions and Recommendations}

Referring on the obtained numerical results and made analysis, it can be deduced that the optimal gap size of the configuration wing-slotted flap is between $1.8 c$ and $3.2 c$, but it cannot be defined exactly on this stage of the study.

As a future work, it is recommended to prepare more numerical simulations and test of the gap size between the pointed above two values. 
Also like a recommendation to confirm the obtained numerical results until now, it can make one numerical measurement of gap size less than $1.8 c$ case and another for a gap size larger than $3.2 c$ case. In the considered cases of the present study, although DES $k-\omega$ SST turbulence model is a good choice for three-dimensional flow field around high-lift configuration, here the application of this model is shown to capture the two-dimensionality of flow physics well.

Because of the relatively good correspondence between experimental data of pure wing airfoil [11] and the configuration with the slotted flap for the obtained aerodynamic characteristics, it supposes the proper choice of methodologies including differencing scheme and grid resolution. Consequently, it can be deduced that the use of DES $k$ - $\omega$ SST hybrid model capture relatively well unsteady flow field around the configuration of wing-slotted flap for 2-D computation.

But in the future work to fulfill the purpose, i.e., optimal gap size, it is recommended the investigation to be focused on the three-dimensional computations. The last recommendation is in order to see if the present numerical approach is applicable for the problems of high-lift system as wing-slotted flap configurations aerodynamics and more specifically optimization of the gap size.

In order to investigate in details the flow field around that type of configuration wing-slotted flap, it is advisable for the next stages of this study to consider the flap deflection.

\section{Acknowledgment}

The authors wish to express their gratitude to $\mathrm{Mr}$.
Ivan Dobrev, Ph.D., Laboratory of Fluids Mechanics, Arts et Metiers ParisTech, Paris, France for his cooperation and help.

\section{References}

[1] Dam, C. P. 2002. "The Aerodynamic Design of Multi-element High-Lift Systems for Transport Airplanes." Progress in Aerospace Sciences 38: 101-44.

[2] G̈unther, B., Thiele, F., Sahner, J., Weinkauf, T., and Hege, H. C. 2007. "Control of Separation on the Flap of a Three-Element High-Lift Configuration." Presented at the 45th AIAA Aerospace Sciences Meeting and Exhibit, Reno, Nevada.

[3] Young, A. D. 1947. "The Aerodynamic Characteristics of Flaps.” R.A.E. Report Aero. 2185.

[4] Cummings, R. M., and Scott, A. M. 2004. "Detached-Eddy Simulation of Slat and Flap Aerodynamics for a High-Lift Wing." Presented at 42nd AIAA Aerospace Sciences Meeting and Exhibit, Reno, Nevada

[5] Silva, F. M., Mattos, B., Fico, N. G. C. R., and Soviero, P. A. O. 2004. "Flap Optimization for Take-off and Landing." Paper CIT04-0229, ABCM, Rio de Janeiro, Brazil.

[6] Foster, D. N., and Irwin, H. P. A. H. 1970 "The Two-Dimensional Flow around a Slotted Flap." Aerodynamic Dept., RAE, Farnborough, Report and Memoranda No. 3681.

[7] Saha, N. 1999. "Gap Size Effect on Low Reynolds Number Wind Tunnel Experiments." Thesis, Aerospace Engineering, Blacksburg, Virginia.

[8] Brezillon, J., Dwight, R. P., and Wild, J. 2008. "Numerical Aerodynamic Optimization of 3D High-Lift Configuration." Presented at the 26th International Congress of the Aeronautical Sciences, Germany.

[9] Ylilammi, N. 2009. "Experimental and Computational Study of Two Flapped Airfoils at Low Reynolds Numbers." Thesis, Helsinki University of Technology.

[10] http://aerospace.illinois.edu/mselig/ads/coord_database.ht $\mathrm{ml}$.

[11] Abbott, I. H., and Von Doenhoff, A. E. 1959. Theory of Wing Sections. USA: NASA. 